\title{
ANÁLISE DA INCIDÊNCIA DAS VARIAÇÕES ANATÔMICAS DOS RAMOS DAS ARTÉRIAS CORONÁRIAS
}

\author{
Ana Carolina Oliveira da Silva ${ }^{\text {* }}$ \\ Leonardo Queiroga Marinho" \\ Lívia Tafnes Almeida de Araújo ${ }^{\text {II }}$ \\ Ruan César Teixeira de Carvalho IV \\ Tânia Regina Ferreira Cavalcanti ${ }^{V}$
}

\begin{abstract}
RESUMO
O estudo morfológico das artérias coronarianas sempre foi um assunto de grande interesse na anatomia e na cardiologia, devido a variabilidade na distribuição das artérias no coração e possíveis correlações anatomoclínicas, entre o tipo de irrigação e o diferente risco de infarto do miocárdio. Dessa forma, com objetivo de abrandar os conhecimentos na área, foi realizada uma pesquisa do tipo experimental, de aspecto descritivo com abordagem e quantitativa no Laboratório de Anatomia da Faculdade de Medicina Nova Esperança, localizado no Bairro Gramame na cidade de João Pessoa - Paraíba. A amostra foi composta por 20 corações cadavéricos dissecados no ano de 2016 e 2017, no referido laboratório. Há muitas variações das artérias coronárias descritas na literatura e que levam em consideração não só a alternância da origem desses vasos, mas também a dominância do suprimento arterial, a diferença do calibre e comprimento quando comparada a maioria. Apesar de uma grande coerência de padrão de trajeto dos corações pesquisados (sendo 90\% regulares), quanto a origem e a ramificação, encontraram-se taxas menores de regularidade (70\%). Ao analisar se poderia haver algum indicativo de correlação entre a origem dos vasos coronarianos e a hipertrofia aparente, notou-se uma pequena taxa de 10\% de correspondência. No entanto, como viés, o número de itens analisados mostrou-se insuficiente para alcançar uma maior fidelidade da anatomia coronariana, além de todas as peças terem como origem comum, o laboratório de anatomia da FAMENE, limitando a qualidade dos resultados.
\end{abstract}

PALAVRAS-CHAVE: Anatomia. Variação Anatômica. Coronária. Cardiologia.

Graduanda do curso de Medicina e Monitores de Correlações Anátomo-clínicas da Faculdade de Medicina Nova Esperança- FAMENE. ORCID: 0000-0001-5716-8136 Autor correspondente: carol_c789@hotmail.com Graduando do curso de Medicina e Monitores de Correlações Anátomo-clínicas da Faculdade de ${ }^{\text {II }}$ Medicina Nova Esperança- FAMENE. ORCID: 0000-0001-5249-6640

Graduanda do curso de Medicina e Monitores de Correlações Anátomo-clínicas da Faculdade de ${ }^{\text {III }}$ Medicina Nova Esperança- FAMENE. ORCID: 0000-0002-9947-2803

Graduando do curso de Medicina e Monitores de Correlações Anátomo-clínicas da Faculdade de ${ }^{I V}$ Medicina Nova Esperança- FAMENE. ORCID: 0000-0003-3180-4223

Professora de Anatomia da Faculdade de Medicina Nova Esperança- FAMENE. IV ORCID: $0000-0003-3084-6720$ 


\section{INTRODUÇÃO}

As artérias coronárias direita e esquerda são os primeiros ramos da aorta e surgem de sua porção ascendente. De enorme relevância para a vida, são responsáveis pela irrigação do coração e pela manutenção de seu adequado funcionamento, contribuindo indiretamente para a nutrição das células de todo o corpo. ${ }^{1}$

As anomalias das artérias coronárias resultam de distúrbios que ocorrem na terceira semana do desenvolvimento fetal. Essas alterações causam variações anatômicas relacionadas a origem, trajetória e término dessas artérias ou a alterações de sua anatomia intrínseca. Algumas anomalias da artéria coronária causam isquemia ocasional, ou obrigatória (origem anômala da coronária esquerda da artéria pulmonar), enquanto outras predispõem a complicações (espasmo, desenvolvimento de placas ateromatosas). ${ }^{2}$

A anatomia desses vasos, universalmente aceita como padrão, é descrita da seguinte forma: os troncos das artérias coronárias se originam dos respectivos seios aórticos anteriores direito e esquerdo e seguem pelo sulco coronário, circundando-o até se encontrarem numa região anatômica denominada cruxcordis. A artéria coronária direita percorre esse sulco emitindo ramos para o átrio e o ventrículo direitos até o ponto de origem da artéria interventricular posterior no chamado padrão de dominância direita (presente em $75 \%$ dos casos). No padrão de dominância esquerda, a artéria interventricular posterior se origina da artéria coronária esquerda, algo que ocorre em $10 \%$ dos casos, ao passo que o padrão de codominância se responsabiliza pelos $15 \%$ restantes. ${ }^{1}$

Todavia, é sabido que há anomalias em menor porcentagem e que merecem atenção científica. Os conceitos de anomalia são muitos e alguns divergem entre si mas, em geral, dizem tratar-se de uma variação do que é encontrado em maior percentagem numa população. No entanto, é possível encontrar na literatura definição de anomalia coronariana como qualquer padrão que tenha uma característica raramente encontrada na população geral. Devem ser considerados os seguintes critérios: é normal qualquer característica morfológica que seja observada em mais de $1 \%$ dos integrantes de uma população; é variante da normalidade uma característica morfológica relativamente incomum, mas prevalente em mais de $1 \%$ dos membros da mesma população; é anomalia uma característica morfológica vista em menos de $1 \%$ da população normal, ou cuja prevalência varia em mais que 02 (dois) desvios-padrão do valor médio em uma curva de distribuição gaussiana $(2,3) \cdot 3$

As anomalias benignas, sem repercussões clínicas importantes para o indivíduo, representam a maioria dos dismorfismos coronarianos. Na artéria coronária esquerda, a variação anatômica mais comum da clássica bifurcação, encontrada em 14-40\% dos casos, é o padrão de trifurcação em que, junto com os ramos interventricular anterior e circunflexo, encontra-se, também, a artéria diagonalis (ou ramo intermédio). Este vaso, quando presente, surge entre os outros dois ramos e supre a parede anterolateral do ventrículo esquerdo. ${ }^{1}$

Cerca de $1 / 3$ de todas as mortes no mundo ocidental é consequência de coronariopatias, e quase todas as pessoas idosas têm algum tipo de distúrbio da circulação arterial coronariana. Por essa razão, nunca é demais enfatizar a anatomia e a fisiologia da circulação coronariana. 4

Portanto, este estudo tem como objetivo analisar as artérias coronárias e suas variações anatômicas em corações do Laboratório 
de Anatomia Humana da Faculdade de Medicina Nova Esperança (FAMENE), no período entre 2016 a 2017, sendo este tema escolhido devido a sua importância no entendimento de

\section{METODOLOGIA}

Foi realizada uma pesquisa do tipo experimental, de aspecto descritivo com abordagem quantitativa no Laboratório de Anatomia da Faculdade de Medicina Nova Esperança, localizado no Bairro Gramame na cidade de João Pessoa-Paraíba. Essa Instituição foi escolhida por disponibilizar de alta quantidade de cadáveres e por ser referência em estudo anatômico em cadáver.

A população para a pesquisa foi composta por todos os corações cadavéricos conservados no laboratório de anatomia da FAMENE. A amostra foi composta por 20 corações cadavéricos dissecados no ano de 2016 e 2017, no referido laboratório. Para seleção da amostra, o critério estabelecido foi as condições de conservação das peças cadavéricas, para assim, criar uma melhor análise das estruturas analisadas. Nesse sentido, foram incluídos corações cadavéricos ainda bem preservados, principalmente no que diz a respeito a sua vascularização, priorizando aqueles ainda recobertos por pericárdio, nos quais não houve manipulação prévia de estruturas internas. Para facilitar a organização das amostras, foram etiquetadas e enumeradas de acordo com o início de sua dissecação.

O instrumento escolhido para a coleta de dados foi um roteiro com questões norteadoras baseadas na anatomia cardíaca descrita na literatura. As questões relacionam-se à vascularização coronariana e suas respectivas ramificações, além de sua dominância, seu comprimento e se o coração é aparentemente hipertrofiado. alterações patológicas, decorrentes de alterações de fluxo sanguíneo, bem como na realização de exames e abordagens cirúrgicas no sistema de irrigação cardíaca.

A coleta de dados foi realizada, após apreciação ética do projeto pelo Comitê de Ética das Faculdades de Enfermagem e Medicina Nova Esperança FACENE/FAMENE, cujo Certificado de Apresentação para Apreciação Ética (CAAE) é 60918416.3.0000.5179. A coleta de dados ocorreu nos meses de dezembro de 2016 a março de 2017. Os dados foram coletados em dias e horários previamente agendados pelo responsável do laboratório de anatomia da FAMENE.

Os dados foram analisados através de método quantitativo. As formas de apresentação dos resultados foram em tabelas, como forma de simplificar o entendimento. Os resultados analisados foram confrontados com a literatura pertinente e, posteriormente, utilizados na confecção do artigo científico.

Foi esperado chegar a uma conclusão de qual perfil anatômico é mais evidente em corações humanos, bem como, problematizar o resultado de forma a esclarecê-lo, realçando sua importância em âmbito regional e levantando possíveis variações anatômicas coronarianas para estudo. 


\section{RESULTADOS E DICUSSÃO}

Durante a dissecação dos 20 corações cadavéricos, no laboratório de anatomia da Faculdade de Medicina Nova Esperança, foi considerado como padrão regular, para trajeto e ramificação, aqueles que se apresentassem de acordo com a disposição vascular mais prevalente na literatura. Os resultados serão expostos nas tabelas 01, 02 e 03.

TABELA 1- Padrão de trajeto coronariano em 20 corações cadavéricos dissecados.

\begin{tabular}{c|c|c}
\hline \hline Padrão de trajeto coronariano & número & $\%$ \\
\hline \hline Padrão regular & 18 & $90 \%$ \\
Padrão irregular & 2 & $10 \%$ \\
\hline \hline TOTAL & 20 & $100 \%$ \\
\hline \hline
\end{tabular}

TABELA 2- Padrão de ramificação coronariana em 20 corações cadavéricos dissecados.

\begin{tabular}{c|c|c}
\hline \hline Padrão de ramificação coronariana & número & $\%$ \\
\hline \hline Padrão regular & 14 & $70 \%$ \\
Padrão irregular & 6 & $30 \%$ \\
\hline \hline TOTAL & 20 & $100 \%$ \\
\hline \hline
\end{tabular}

TABELA 3- Origens incomuns das artérias coronárias.

\begin{tabular}{|c|c|c|}
\hline Padrão de origem & número & $\%$ \\
\hline Padrão regular & 14 & $70 \%$ \\
\hline Padrão irregular & 6 & $30 \%$ \\
\hline TOTAL & 20 & $100 \%$ \\
\hline
\end{tabular}

A anatomia coronariana normal, universalmente aceita, é assim definida: o tronco coronariano origina-se do seio coronariano esquerdo e divide-se na artéria descendente anterior (ou interventricular anterior) e na artéria circunflexa. A artéria descendente anterior segue posteriormente ao tronco pulmonar no sulco interventricular anterior; a artéria circunflexa segue no sulco atrioventricular posterior; a artéria coronária direita origina-se do seio coronariano direito e segue pelo sulco atrioventricular anterior. 5,6. A figura 1 repre- senta bem.

A alternância da origem dos vasos coronarianos é de extrema relevância, pois a origem e o trajeto proximal das artérias coronárias anômalas são os principais fatores preditivos de gravidade para acometimento da musculatura cardíaca.7 Todavia, o diagnóstico precoce dificilmente ocorre, sendo a maioria dos casos, diagnosticados de forma incidentalmente ou em necropsias, pois as anomalias coronarianas congênitas são geralmente assintomáticos. Dessa forma, consiste na se- 
gunda causa mais frequente de morte súbita

cárdica, especialmente em adultos jovens. 9 cardíaca, ou outros sintomas de isquemia mio-

FIGURA 1 - Ilustração de vascularização coronária mais comum.

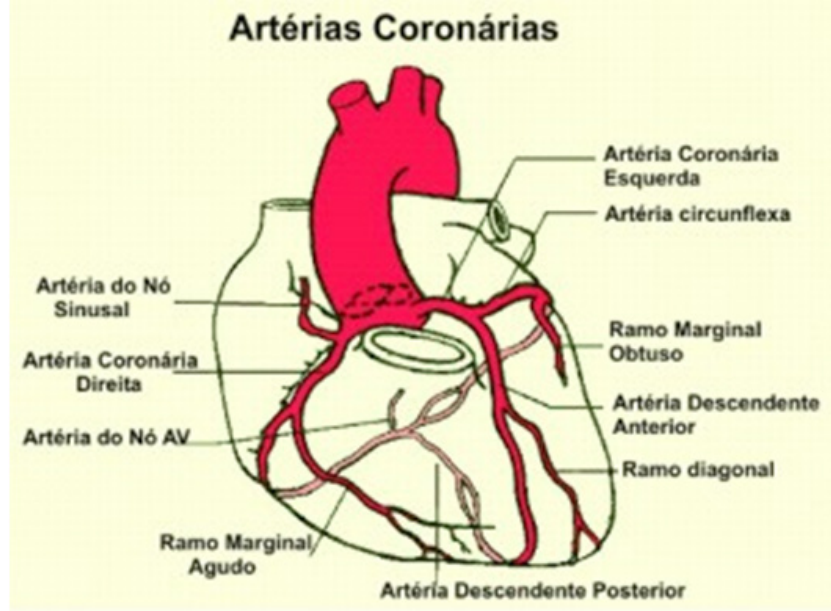

Na figura 2 é possível observar um coração dissecado em que o padrão de trajeto e origem se apresenta regular.

Em longo prazo, a irrigação inadequada do miocárdio pode levar a falência da funcionalidade da fibra muscular cardíaca, o que pode gerar uma cardiomiopatia dilatada ou hipertrófica quando o coração necessita de mais trabalho para ejetar o sangue para fora da câmera. Dessa forma, foi analisada a correlação entre a origem incomum dos vasos coronarianos e a presença de hipertrofia aparente, conforme exposto na tabela 04.

FIGURA 2 - Exemplo de coração dissecado com vascularização em padrão mais comum.

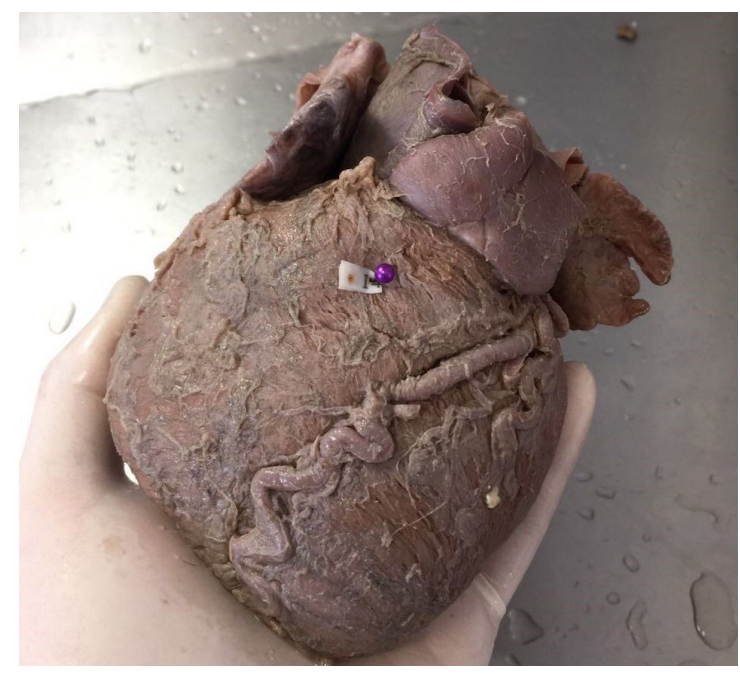

Rev. Nova Esperança. 2019; 17(1): 53-61 
TABELA 4 - Correlação entre hipertrofia e origens incomuns das artérias coronárias.

\begin{tabular}{lc}
\hline \hline Correlações & Número \\
\hline \hline Coração com hipertrofia aparente e o com origens coronarianas incomuns & 2 \\
Coração com hipertrofia aparente e sem origens coronarianas incomuns & 5 \\
Coração sem hipertrofia aparente e com origens coronarianas incomuns & 9 \\
Coração sem hipertrofia aparente e sem origens coronárias incomuns & 4 \\
\hline \hline TOTAL & 20 \\
\hline \hline
\end{tabular}

É sabido que para melhor conclusão da correção entre a hipertrofia cardíaca e as origens incomuns das artérias coronarianas é necessário um estudo mais detalhado. Todavia, de acordo com o presente estudo, não houve correlação aparente, pois a maioria dos corações, visivelmente hipertrofiados a "olho nu", não possuíam origens coronárias incomuns, levando a conclusão de hipertrofia por outras causas que não a vascular.

Há muitas variações das artérias coronárias descritas na literatura e que levam em consideração não só a alternância da origem desses vasos, mas também, a dominância do suprimento arterial, a diferença do calibre e comprimento quando comparada a maioria.

Uma das variações importantes que pode acontecer é com o ramo interventricular posterior, o qual determina os tipos de supri- mentos das paredes do coração. Em 55\% dos casos, o ramo interventricular posterior se origina da artéria coronária direita, mas não envolve a face posterior do ventrículo esquerdo. Já em $25 \%$ dos casos, o ramo interventricular posterior é derivado da artéria coronária esquerda e ainda, em $20 \%$ dos casos, a artéria coronária direita não origina somente o ramo interventricular posterior, mas também supre partes da face posterior do ventrículo esquerdo. Sendo assim, é predominante a dominância balanceada, na qual o ramo interventricular posterior sai da artéria coronária direita, mas não emite ramos para a face posterior do ventrículo esquerdo.10 No estudo, pós dissecação dos corações no laboratório de anatomia, foram encontrados os dados abordados na tabela 05.

TABELA 5 - Dominância vascular observada nos corações dissecados no laboratório de anatomia da Faculdade de Medicina Nova Esperança.

\begin{tabular}{lcc}
\hline \hline Dominância vascular coronariana & número & $\%$ \\
\hline \hline Dominância de direita & 12 & $60 \%$ \\
Dominância balanceada & 7 & $35 \%$ \\
Dominância de esquerda & 1 & $5 \%$ \\
\hline \hline TOTAL & 20 & $100 \%$ \\
\hline \hline
\end{tabular}

De acordo com a tabela, houve um predomínio da dominância de direita, o que vai de encontro com o proposto na literatura. ${ }^{10}$
No entanto, em outro estudo, no qual se fez a relação entre o padrão anatômico e as condições patológicas das artérias coronárias, 
em 269 corações humanos encontra dominância de direita em $48 \%$, balanceada, em $34 \%$, e dominância de esquerda em $18 \%$. Refere, ain$\mathrm{da}$, que os infartos, ocorridos nos corações com circulação balanceada, são mais benignos, raramente levando a morte, concluindo que o tipo de circulação balanceada é o meIhor padrão para o ser humano. ${ }^{11}$ Por outro lado, os corações com dominância de esquerda constituem um grupo patologicamente importante, pois são os que mais sofrem com a arteriosclerose e, consequentemente, com o infarto do miocárdio. A figura 3 mostra um dos corações com dominância balanceada.

Como pôde ser observado, vários autores destacam a importância do conhecimento das mais diversas variações da irrigação arterial cardíaca, sendo interessante, portanto, ao acadêmico de medicina, aprender sobre as principais variações e os mais relevantes aspectos clínicos que essas trazem consigo.

FIGURA 3 - Coração dissecado com dominância balanceada.

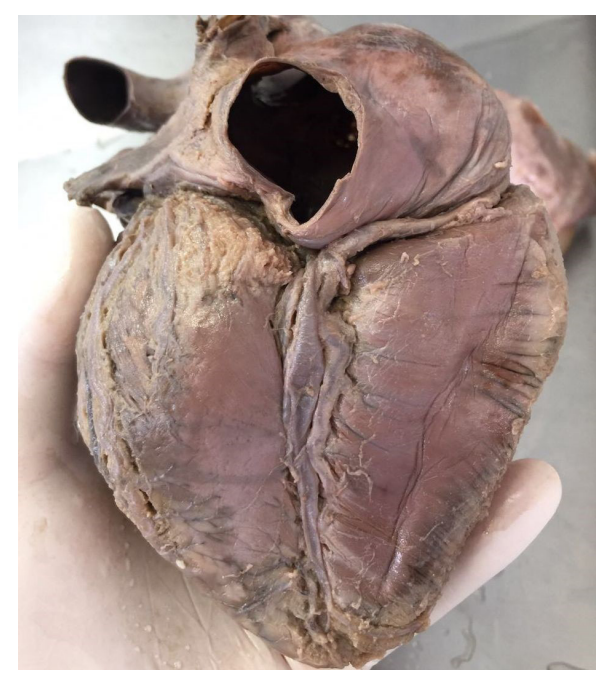

\section{CONCLUSÃO}

Findado o estudo, pode-se concluir que a anatomia coronariana verdadeiramente é muito diversa. Apesar de uma grande coerência de padrão de trajeto dos corações pesquisados (sendo $90 \%$ regulares), quanto a origem e a ramificação, encontraram-se taxas menores de regularidade (70\%).

Ao analisar se poderia haver algum indicativo de correlação entre a origem dos vasos coronarianos e a hipertrofia aparente, notou-se uma pequena taxa de $10 \%$ de correspondência.

A dominância mais encontrada foi a direita (60\%), prevalência $25 \%$ maior que na literatura pesquisada; a dominância mista obteve percentualmente valor próximo (35\% no presente trabalho, contra 34\%); e a dominância esquerda foi encontrada em apenas 1 coração, representando $5 \%$, bem abaixo dos $18 \%$ do trabalho supracitado. 
Fatores como a região geográfica pesquisada, à época, o número de corações estudados podem ser a explicação para algumas diferenças encontradas em relação a outros trabalhos publicados. No entanto, como viés, o número de itens analisados (20 corações) mostrou-se insuficiente para alcançar uma maior fidelidade da anatomia coronariana, além de todas as peças possuírem como origem o mesmo local, o laboratório de anatomia da FAMENE, limitando, assim, a qualidade dos resultados.

\title{
ANALYSIS OF THE INCIDENCE OF ANATOMICAL VARIATIONS OF THE CORONARY ARTERIES SEGMENTS
}

\begin{abstract}
The morphological study of the coronary arteries has always been a subject of great interest in anatomy and cardiology. Due to the variability in the distribution of the arteries in the heart and possible anatomic-clinical correlations between the type of irrigation and the different risk of myocardial infarction, the coronary arteries have been better studied lately, with the diffusion of the coronary angiography. A research of the experimental type, of descriptive aspect with an approach and quantitative in the Anatomy Laboratory of Nova Esperança Medical School, located in Bairro Gramame in the city of João Pessoa - Paraíba. The sample consisted of 20 cadaveric hearts dissected in the year 2016 and 2017, in mentioned laboratory. There are many variations of the coronary arteries described in the literature and that consider not only the alternation of the origin of these vessels, but also the dominance of the arterial supply, the difference of the gauge and length when compared to the majority. Despite a great consistency of path pattern of the hearts studied (being $90 \%$ regular), as to origin and branching, we found lower rates of regularity (70\%). When analyzing whether there could be any indication of correlation between the origin of coronary vessels and apparent hypertrophy, a small $10 \%$ correspondence rate was noted. However, as a bias, the number of analyzed items proved to be insufficient to achieve greater fidelity of the coronary anatomy, in addition to all the pieces having a common origin, the FAMENE anatomy laboratory, limiting the quality of the results.
\end{abstract}

KEYWORDS: Anatomy. Anatomical Variation. Coronary. Cardiology.

\section{REFERÊNCIAS}

1. Souza Batista AV, Porto EA, Molina GP. Estudo da anatomia da artéria coronária esquerda e suas variações: perspectivas de nova classificação. Revista Saúde \& Ciência Online. 2011;2(1):5565.

2. Farias DC, Moreira AC, Tavares JM, Correia JN, Souza RS, Silva-Filho AR. Origem anômala da ar- téria coronária esquerda do seio de Valsalva direito. Rev. bras. cardiol. invasiva. 2013;21(1):82-4.

3. Angelini P, Velasco JA, Flamm S. Coronary anomalies: incidence, pathophysiology, and clinical relevance. Circulation. 2002 May 21;105(20):244954. 
4. Souza SC. Lições de anatomia: manual de esplancnologia. 1. ed. Salvador: EDUFBA; 2010.

5. Shi H, Aschoff AJ, Brambs HJ, Hoffmann $\mathrm{MH}$. Multislice $\mathrm{CT}$ imaging of anomalous COronary arteries. Europeanradiology. 2004 Dec 1;14(12):2172-81.

6. Taylor AM, Thorne SA, Rubens MB, Jhooti P, Keegan J, Gatehouse PD, Wiesmann F, Grothues F, Somerville J, Pennell DJ. Coronary artery imaging in grown up congenital heart disease: complementary role of magnetic resonance and x-ray coronary angiography. Circulation. 2000 Apr 11;101(14):1670-8.

7. Veras FH, Victor EG, Saraiva LC, Lopes MM. Origem anômala das artérias coronárias. Rev Bras Cardiollnvas. 2007 Sep;15(3):285-92.
8. Zeina AR, Blinder J, Sharif D, Rosenschein U, Barmeir E. Congenital coronary artery anomalies in adults: non-invasive assessment with multidetector CT. The British journalofradiology. 2009 Mar;82(975):254-61.

9. Takimura CH, Nakamoto A, Hotta VT, Campos MF, Málamo M, Otsubo R. Origem anômala da artéria coronária esquerda no tronco pulmonar. relato de um caso em adulto. ArqBrasCardiol. 2002;78(3):309-14.

10. Sobotta J et al.. Sobotta atlas de anatomia humana. 23. ed. Rio de Janeiro: Guanabara Koogan, 2012. $3 \mathrm{v}$.

11. Schlesinger MJ. Significant variations in the anatomic pattern of the coronary vessels. Blood Heart Circ. 1940;13:93-7. 